\title{
Amplitude Modulation Response and Linearity Improvement of Directly Modulated Lasers Using Ultra-Strong Injection-Locked Gain-Lever Distributed Bragg Reflector Lasers
}

\author{
Hyuk-Kee Sung* \\ School of Electronic and Electrical Engineering, Hongik University, Seoul 121-791, Korea \\ Ming C. Wu \\ Department of Electrical Engineering and Computer Sciences, University of California at Berkeley, \\ CA 94720 USA
}

(Received November 12, 2008 : revised December 5, 2008 : accepted December 8, 2008)

\begin{abstract}
Directly modulated fiber-optic links generally suffer higher link loss and larger signal distortion than externally modulated links. These result from the electron-photon conversion loss and laser modulation dynamics. As a method to overcome the drawbacks, we have experimentally demonstrated the RF performance of directly modulated, ultra-strong injection-locked gain-lever distributed Bragg reflector (DBR) lasers. The free-running DBR lasers exhibit an improved amplitude modulation efficiency of $12.4 \mathrm{~dB}$ under gain-lever modulation at the expense of linearity. By combining gain-lever modulation with ultra-strong optical injection locking, we can gain the benefits of both improved modulation efficiency from the gain-lever effect, plus improved linearity from injection locking. Using an injection ratio of $R=11 \mathrm{~dB}$, a 23.4-dB improvement in amplitude response and an 18-dB improvement in spurious-free dynamic range have been achieved.
\end{abstract}

Keywords : Optical injection locking, Directly modulated semiconductor laser, Gain-lever modulation

OCIS codes : (140.5960) Semiconductor lasers; (140.3520) Lasers, injection-locked

\section{INTRODUCTION}

Direct modulation of semiconductor lasers has been widely investigated in analog fiber-optic applications such as cable television distribution systems, antenna remoting in cellular networks, and phased arrayed antennas [1]. In digital links, 40-Gb/s transmission through a directly modulated link has been successfully demonstrated [2]. The requirements for analog and digital signal transmissions are quite different. Low radio-frequency (RF) link loss and small nonlinear distortions are needed to achieve good analog performance, while high modulation bandwidth, low chirp and high extinction ratios are important for digital links [3, 4]. Link loss in directly modulated links is mainly due to the electrical-to-optical conversion process in lasers.

*Corresponding author: hksung@hongik.ac.kr
Several approaches have been proposed to increase the modulation efficiency of semiconductor lasers, including gain-lever modulation [5-7] and cascaded lasers [8-11]. Cascaded lasers achieve higher efficiency by recycling the RF modulating current through multiple lasers connected in series. First proposed using discrete lasers [8], a serial connection of six discrete lasers with a link gain of $+3.78 \mathrm{~dB}$ was demonstrated. However, the measured link bandwidth was about $60 \mathrm{MHz}$, whereas the $3-\mathrm{dB}$ bandwidth of the individual lasers was greater than $3 \mathrm{GHz}$. This was due to the parasitics of the series-connected lasers. To realize a bandwidth closer to the individual components, monolithically-integrated versions were proposed using surface-emitting [9] and edge-emitting lasers $[10,11]$. Improved link performance exhibiting $>100 \%$ differential efficiency and a spuriousfree dynamic range (SFDR) of $120 \mathrm{~dB} \cdot \mathrm{Hz}^{2 / 3}$ operating at $500 \mathrm{MHz}$ has been demonstrated [10]. 


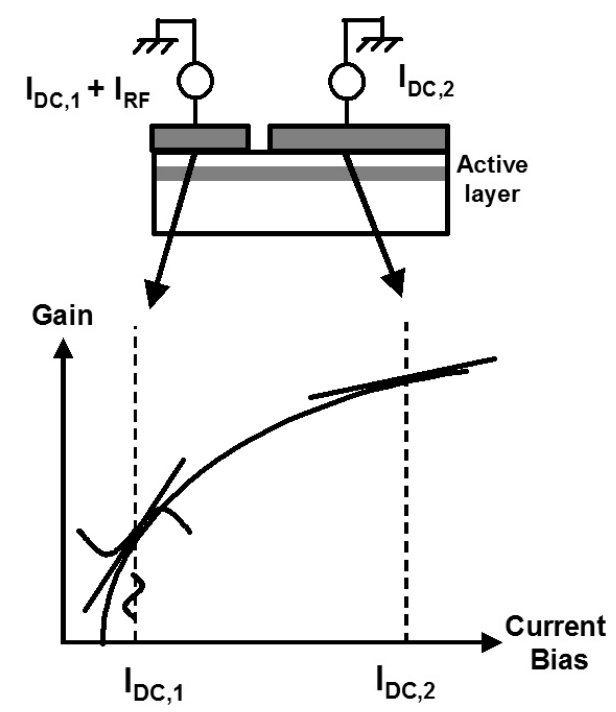

FIG. 1. Gain-lever effect in semiconductor lasers, after [3].

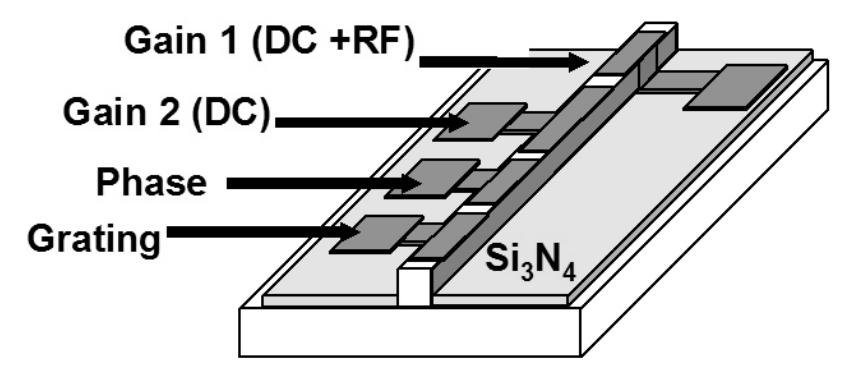

FIG. 2. Schematic of distributed Bragg reflector (DBR) laser for gain-lever modulation.

Among the techniques, gain-lever lasers are structurally simple and directly compatible with the standard fabrication process of conventional lasers. Gain-lever lasers take advantage of the nonlinear gain-versus-current characteristics in quantum-well gain media. Figure 1 illustrates the principle of gain-lever modulation. GaAlAs/GaAs single quantum well $[5,6]$ and $\mathrm{InGaAsP} / \mathrm{InP}$ multiple quantum well [7] gain-lever lasers have been demonstrated. Unfortunately, the improved amplitude modulation (AM) efficiency is obtained at the expense of linearity. Furthermore, the previous gain-lever devices were FabryPerot lasers operating in multiple longitudinal modes, and are not suitable for practical system applications.

Strong optical injection locking is a promising technique to suppress the nonlinear distortion as well as increase the resonance frequency of directly modulated semiconductor lasers [12-17]. The nonlinear distortions are more pronounced near the relaxation oscillation frequency of the laser due to the nonlinear coupling between electrons, photons, and phase difference between master and slave. By pushing the resonance to frequencies much higher than the signal band, the nonlinear distortions can be significantly reduced. This has been successfully demonstrated in directly modulated links using distributed feedback lasers [14] and vertical-cavity surface-emitting lasers [16]. In addition, the frequency chirp and the relative intensity noise are also reduced in injectionlocked lasers [12, 18].

Recently, we successfully demonstrated an improvement in RF performance by injection locking gain-levermodulated distributed Bragg grating (DBR) lasers [19]. In this paper, we provide a detailed characterization of the gain-lever modulation and further performance enhancement by ultra-strong injection locking (injection ratio $R=11 \mathrm{~dB}$ ) on top of the previously reported injection locking of gain-lever DBR lasers. This combination provides simultaneous enhancement of modulation efficiency as well as a reduction of nonlinear distortions. With gain-lever modulation and ultra-strong optical injection locking, a 23.4-dB increase in AM efficiency (12.4 $\mathrm{dB}$ by gain-lever modulation and $11 \mathrm{~dB}$ by ultrastrong optical injection) and 18-dB enhancement of SFDR have been demonstrated.

\section{EXPERIMENTAL RESULTS}

Figure 2 shows the device schematic of the gain-lever DBR laser. The gain-lever DBR laser consists of four sections: a grating, phase, and two electrically-isolated gain sections. The grating and phase sections are designed to perform coarse and fine wavelength tuning with single-mode operation. Various split ratios, defined as the length of the shorter gain section divided by the total length of the gain sections, are used to investigate the optimum gain-lever geometry. The laser is designed with a capped-mesa buried heterostructure. After epitaxial growth of the capped-mesa buried heterostructure, a ridge height of $\sim 3-4-\mu \mathrm{m}$ is formed to reduce parasitic capacitance. A 500-nm layer of silicon nitride passivates the surface. P-metal contacts for the grating, phase, and gain sections are formed using $\mathrm{Ti} / \mathrm{Pt} / \mathrm{Au}$. The bottom n-contact is comprised of $\mathrm{Au} / \mathrm{Sn} / \mathrm{Au}$. The contact resistance obtained was typically less than $10 \Omega$ after annealing. A final, $0.5-\mu \mathrm{m}$ deep isolation etch is performed to increase the electrical isolation among the sections. The resistance between the adjacent sections is greater than $4 \mathrm{k} \Omega$.

Figure 3 shows the experimental setup for measuring the performance of the gain-lever DBR laser, with and without optical injection locking. A gain-lever DBR laser with a split ratio of 0.5 is used for all the measurements presented in this paper. A tunable laser with an external cavity is used as the master laser. The output of the master laser is amplified by an Erbium-doped fiber amplifier to achieve ultra-strong injection locking. An inline optical attenuator controls the injection power. A polarization controller and an optical circulator with $>40-\mathrm{dB}$ isolation complete the master laser module. The output of the gain-lever DBR laser is monitored by a 


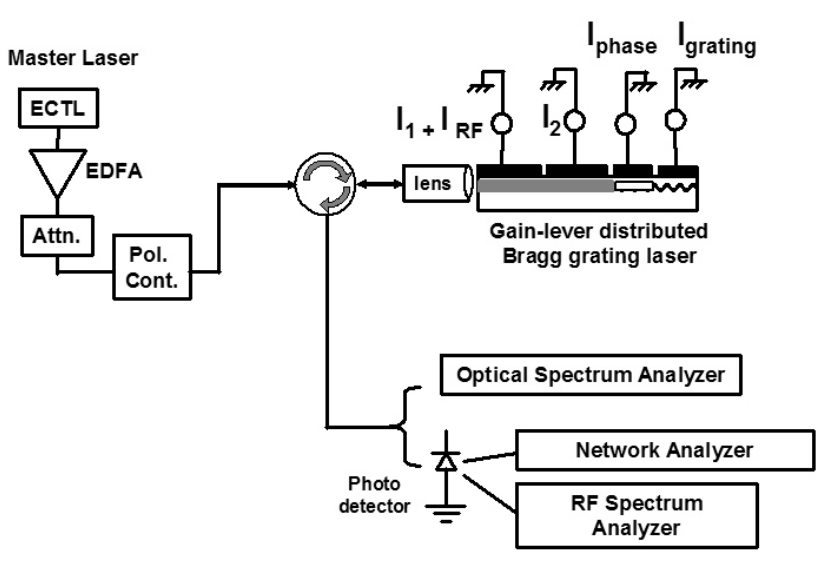

FIG. 3. Experimental setup for measuring RF performance of a gain-lever DBR laser with and without injection locking. (ECTL: external cavity tunable laser; EDFA: Erbium-doped fiber amplifier; Attn.: optical attenuator; Pol. cont.: polarization controller).

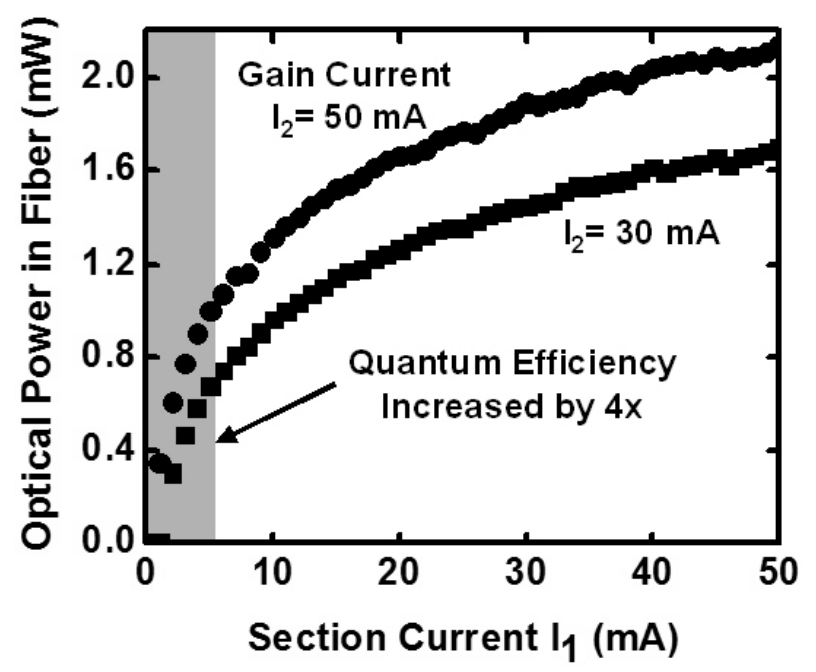

FIG. 4. Measured optical power versus the bias current on section showing the effect of gain-lever modulation.

high-resolution (0.01 $\mathrm{nm})$ optical spectrum analyzer. To observe the RF modulation characteristics such as frequency response, nonlinear distortions, and SFDR, a $\mathrm{DC}$ bias $\left(\mathrm{I}_{1}\right)$ and $\mathrm{RF}$ signal $\left(\mathrm{I}_{\mathrm{RF}}\right)$ are applied to one of the gain sections, while the other gain section is biased at a constant DC current $\left(\mathrm{I}_{2}\right)$. The modulated output is coupled to a high-speed (34-GHz) photodetector and monitored by a network analyzer or RF spectrum analyzer.

The DC light-versus-current curves are shown in Fig. 4. The current $\mathrm{I}_{2}$ (see Fig. 3 for the definition of current symbols) is fixed either at 30 or $50 \mathrm{~mA}$, while current $I_{1}$ is varied from 0 to $50 \mathrm{~mA}$. As depicted in the shaded area in Fig. 4, a four-fold increase of the quantum efficiency (equivalent to an RF efficiency increase of $12 \mathrm{~dB}$ ) is achieved when the RF modulation section (=section 1$)$ is biased at a low level $(<5 \mathrm{~mA})$.

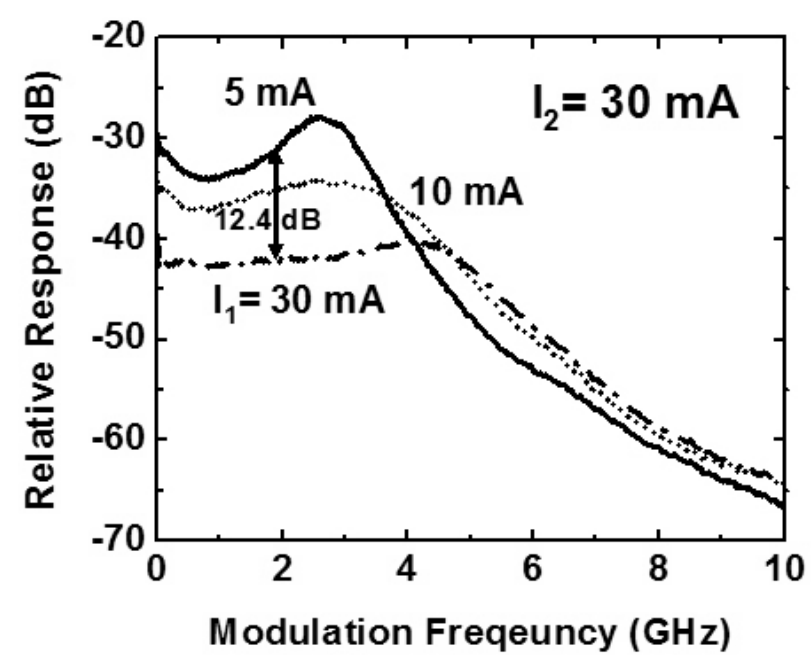

FIG. 5. Measured modulation responses of the gain-lever DBR laser for various operating conditions.

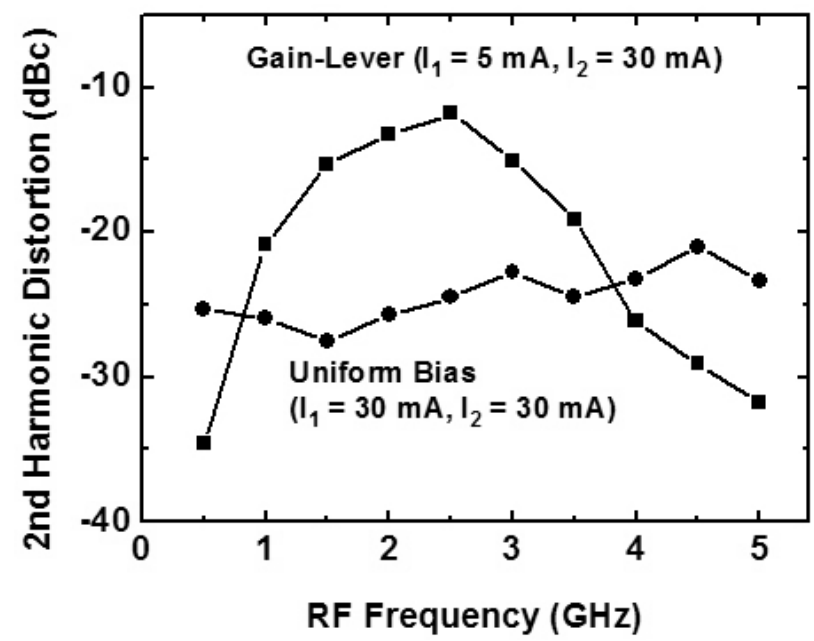

FIG. 6. The second harmonic distortion versus modulation frequency for gain-lever and uniform bias condition. The second harmonic product is measured at twice the RF modulation frequency.

The modulation response of the gain-lever DBR laser is shown in Fig. 5. As the bias current on the modulation section (section 1) decreases, the differential gain increases, producing a higher modulation response. Compared with the uniformly biased condition $\left(\mathrm{I}_{1}=30 \mathrm{~mA}, \mathrm{I}_{2}=30\right.$ $\mathrm{mA}$ ), a 12.4-dB improvement in modulation efficiency has been achieved by the gain-lever modulation $\left(\mathrm{I}_{1}=5\right.$ $\mathrm{mA}, \mathrm{I}_{2}=30 \mathrm{~mA}$ ). However, as a consequence of the low current bias necessary for gain-lever modulation, the modulation bandwidth decreases and nonlinear distortion increases. The resonance frequency of the laser is decreased from $5 \mathrm{GHz}$ to $3 \mathrm{GHz}$. The measured second harmonic distortion as a function of modulation frequency is shown in Fig. 6. The laser is modulated with a $2-\mathrm{GHz}$ $\mathrm{RF}$ signal. The free-running gain-lever laser $\left(\mathrm{I}_{1}=5 \mathrm{~mA}\right.$, $\mathrm{I}_{2}=30 \mathrm{~mA}$ ) shows a severe distortion of $-10.5 \mathrm{dBc}$ at 


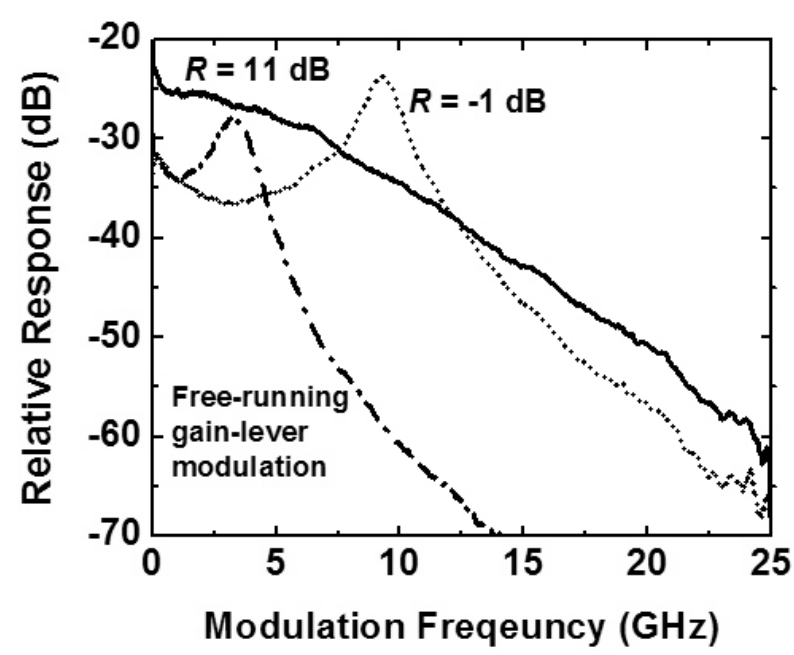

FIG. 7. Measured frequency responses of a free-running, strong $(R=-1 \mathrm{~dB}, \Delta f=-7.4 \mathrm{GHz})$, and ultra-strong $(R=$ $11 \mathrm{~dB}, \Delta f=-32 \mathrm{GHz})$ injection-locked DBR laser with gain-lever modulation.

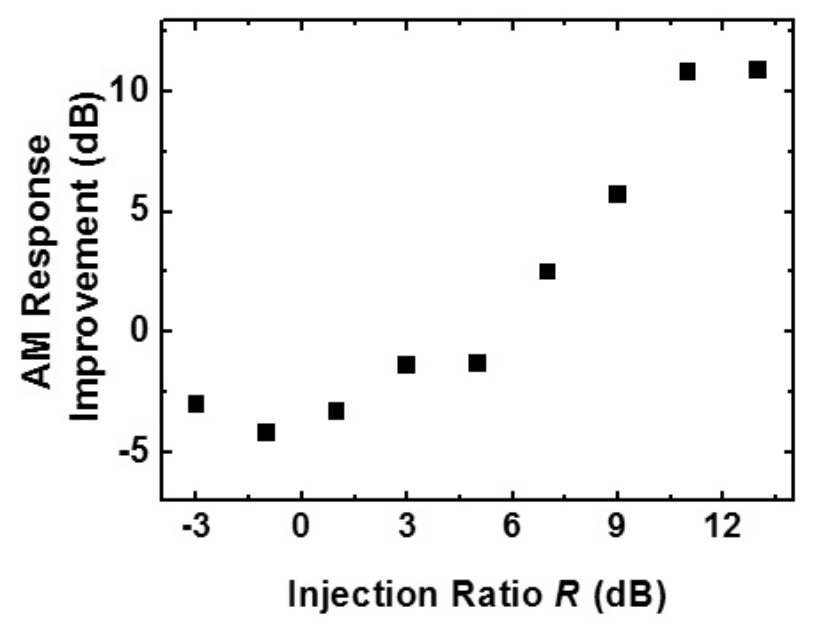

FIG. 8. Measured AM response improvement for various injection ratios. Frequency detuning values are adjusted for the corresponding injection ratios to achieve the largest AM improvement.

2.5- $\mathrm{GHz}$ modulation; $15-\mathrm{dB}$ higher than that of the uniform bias condition. This is due to laser nonlinearity originating from both nonlinear light-versus-current curves of the gain-lever modulation (Fig. 4) and the laser resonance peak at $\sim 3 \mathrm{GHz}$ (Fig. 5).

Figure 7 shows the measured frequency responses of the gain-lever laser under free-running, strong injectionlocking $(R=-1 \mathrm{~dB}, \Delta f=-7.4 \mathrm{GHz})$, and ultra-strong injection-locking $(\mathrm{R}=11 d B, \Delta f=-32 \mathrm{GHz})$ conditions. The frequency detuning, $\Delta f$, is defined as the frequency difference between the master and the free-running slave lasers $\left(\Delta f=f_{\text {master }}-f_{\text {free,slave }}\right)$. Injection ratio, $\mathrm{R}$, is defined as the power ratio between the injected power and the lasing power of the free-running slave laser inside the

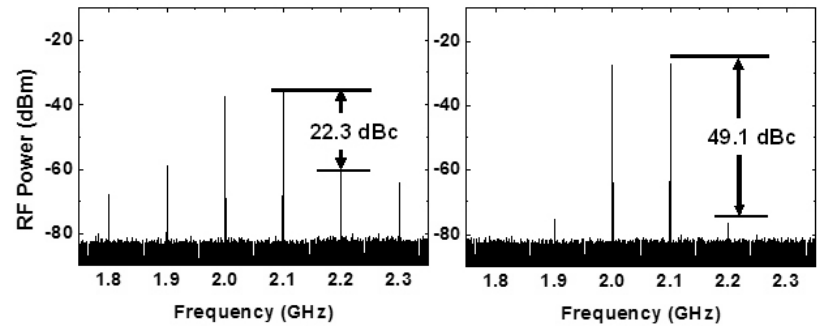

FIG. 9. Measured RF spectra showing the two-tone intermodulation distortions $\left(f_{1}=2.0 \mathrm{GHz}, f_{2}=2.1 \mathrm{GHz}\right)$ of the gain-lever DBR laser under (a) free-running and (b) ultra strong injection-locking conditions $(R=11 \mathrm{~dB}, \Delta$ $f=-32 \mathrm{GHz})$.

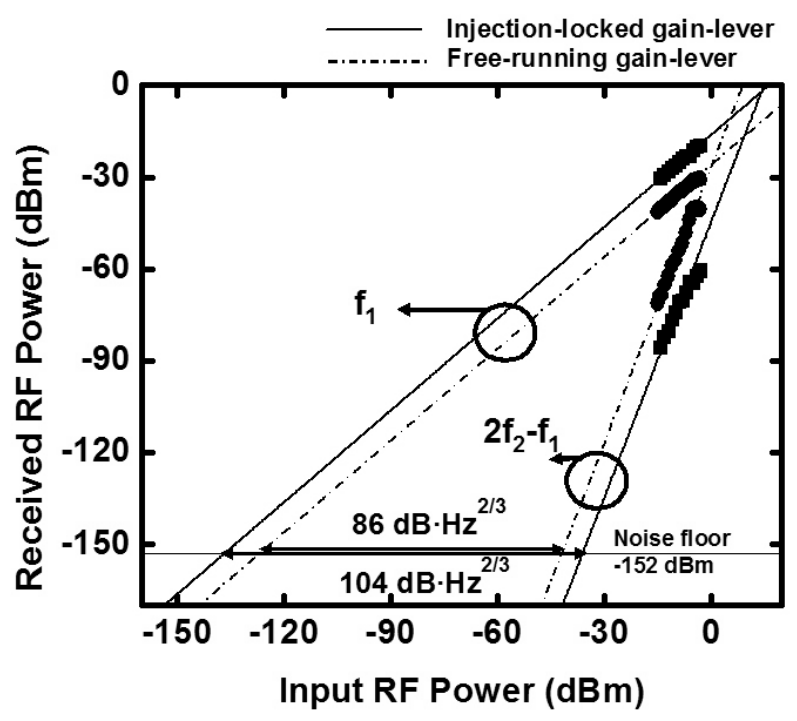

FIG. 10. Measured SFDR of the link with a directly modulated gain-lever DBR laser $\left(f_{1}=2.0 \mathrm{GHz}, f_{2}=2.1 \mathrm{GHz}\right)$.

laser cavity. When the gain-lever DBR laser is injectionlocked with $R=-1 \mathrm{~dB}$, the resonance frequency is increased to $8.7 \mathrm{GHz}$ without severely compromising the enhanced AM efficiency of the gain-lever modulation. Under a high injection level of $R=11 \mathrm{~dB}$, the AM efficiency is enhanced by $11 \mathrm{~dB}$ around $2 \mathrm{GHz}$. Frequency detuning is optimized to achieve the highest modulation efficiency under the injection ratio of $R=11 \mathrm{~dB}$. The improvement of the AM efficiency is due to a cavity resonance effect causing an amplitude response increase at a low frequency band, combined with the increased optical power under ultra-strong injection. In optical injection locking system, the role of the modulated slave laser is similar to the conventional optical modulator. However, the modulation response of the slave laser exhibits significantly different characteristics from the conventional optical modulator by tuning the two injection locking parameters, $\Delta f$ and $\mathrm{R}$ [17]. We have utilized the large negative detuning regime for the AM response improvement by the injectionlocked laser on top of the improvement by the gain-lever 
modulation.

Figure 8 shows the dependence of the AM response improvement under 2-GHz $\mathrm{RF}$ modulation for various injection ratios. The improvement is calculated by dividing $\mathrm{RF}$ power at $2-\mathrm{GHz}$ under a given injection ratio with the value of free-running gain-lever modulation. Frequency detuning values are adjusted for the corresponding injection ratios to achieve the largest $\mathrm{AM}$ improvement. The gain-lever laser is biased with $\mathrm{I}_{1}=5$ $\mathrm{mA}$ and $\mathrm{I}_{2}=30 \mathrm{~mA}$. It is modulated by a single-tone RF signal on section $1(f=2 \mathrm{GHz}$, modulation power $=-10 \mathrm{dBm}$ ). The received $\mathrm{RF}$ power at $2 \mathrm{GHz}$ is measured and normalized by that of the free-running gain-lever state. The AM response improvement reaches its maximum with the ultra-strong injection ratio of $\sim 11 \mathrm{~dB}$. The largest improvement of $11 \mathrm{~dB}$ is achieved with the injection ratio of $11 \mathrm{~dB}$, which is a 23.4- $\mathrm{dB}$ improvement over the free-running uniform bias case. No more improvement is observed with larger than $12-\mathrm{dB}$ injection ratio due to the saturation of the photodetector.

The third-order intermodulation distortion (IMD3) is measured by a two-tone RF modulation signal $\left(f_{1}=\right.$ $2.0 \mathrm{GHz}, f_{2}=2.1 \mathrm{GHz}$, modulation power $\left.=-10 \mathrm{dBm}\right)$. As shown in Fig. 9, with ultra-strong injection locking $(R=11 \mathrm{~dB}$ and $\Delta f=-32 \mathrm{GHz})$, the fundamental tones at $2.0 \mathrm{GHz}$ and $2.1 \mathrm{GHz}$ are increased by $11 \mathrm{~dB}$ above the free-running gain-lever modulation tones. The IMD3 for the free-running laser is $-22.3 \mathrm{dBc}$. In comparison, the IMD3 of the injection-locked state is reduced considerably to $-49.1 \mathrm{dBc}$, exhibiting a $26.8-\mathrm{dB}$ reduction in IMD3 compared with the free-running gain-lever modulation.

An important figure of merit of analog fiber-optic links is SFDR. Figure 10 shows the received RF powers of the fundamental and the third-order inter-modulation product versus the input RF power for the free-running gain-lever and ultra-strong injection-locked gain-lever conditions $(R=11 \mathrm{~dB}$ and $\Delta f=-32 \mathrm{GHz})$. The laser is modulated with a two-tone $\mathrm{RF}$ signal $\left(f_{1}=2.0 \mathrm{GHz}\right.$, $f_{2}=2.1 \mathrm{GHz}$ ). The SFDR of the injection-locked gainlever DBR laser is enhanced by $18 \mathrm{~dB}$ compared with the free-running gain-lever modulation: $11 \mathrm{~dB}$ by the increase of amplitude response and $7 \mathrm{~dB}$ by the reduction of the third-order inter-modulation product.

\section{CONCLUSION}

The ultra-strong optically injection-locked gain-lever distributed Bragg reflector laser has been successfully demonstrated. Gain-lever modulation improves the amplitude modulation efficiency by $12.4 \mathrm{~dB}$, at the expense of linearity. By combining the gain-lever modulation with ultra-strong optical injection locking with an 11$\mathrm{dB}$ injection ratio, the amplitude response is increased by $11 \mathrm{~dB}$ above the gain-lever improvement and the third-order inter-modulation distortion has been suppressed by $26.8 \mathrm{~dB}$. This results in an 18-dB improvement in spurious-free dynamic range. This new modulation scheme can improve the link loss, bandwidth and signal fidelity of directly modulated fiber-optic links.

\section{ACKNOWLEDGMENT}

The authors would like to thank Dr. K. Y. Liou in Multiplex Inc. and Erwin K. Lau at University of California, Berkeley for their helpful discussion. This work was supported by the Hongik University new faculty research support fund.

\section{REFERENCES}

[1] C. Cox, III, E. Ackerman, R. Helkey, and G. E. Betts, "Direct-detection analog optical links," IEEE Trans. Microw. Theory Tech., vol. 45, no. 8, pp. 1375-1383, Aug. 1997.

[2] K. Sato, S. Kuwahara, Y. Miyamoto, and N. Shimizu, "40 Gbit/s direct modulation of distributed feedback laser for very-short-reach optical links," Electron. Lett., vol. 38, no. 15 , pp. 816-817, Jul. 2002.

[3] I. M. Jung and D. -S. Shin, "Efficient Optical Intensity Modulator Based on the Electrically Tunable LiNbO3 Reflection Grating for Analog Fiber-Optic Links," $J$. Opt. Soc. Korea, vol. 11, no. 1, pp. 1-5, Mar. 2007.

[4] M. -K. Hong, S. -K. Han, and S. -H Lee, "Linearization of DFB LD by using Cross Gain Modulation of Reflective SOA in Radio-over-Fiber Link," J. Opt. Soc. Korea, vol. 11, no. 4, pp. 158-161, Dec. 2007.

[5] N. Moore and K. Y. Lau, "Ultrahigh efficiency microwave signal transmission using tandem-contact single quantum well GaAlAs lasers," Appl. Phys. Lett., vol. 55, no. 10, pp. 936-938, Sep. 1989.

[6] K. J. Vahala and M. A. Newkirk, "The optical gain lever: A novel gain mechanism in the direct modulation of quantum well semiconductor lasers," Appl. Phys. Lett., vol. 54, no. 25, pp. 2506-2508, Jun. 1989.

[7] C. P. Seltzer, L. D. Westbrook, and H. J. Wickes, "The "gain-lever" effect in InGaAsP/InP multiple quantum well lasers," J. Lightwave. Tech., vol. 13, no. 2, pp. 283289, Feb. 1995.

[8] C. H. Cox, III, H. V. Roussell, R. J. Ram, and R. J. Helkey, "Broadband, directly modulated analog fiber link with positive intrinsic gain and reduced noise figure," International Topical Meeting on Microwave Photonics (MWP), pp. 157-160, Oct. 1998.

[9] R. J. Ram, F. Rana, and P. Mayer, "Cascade semiconductor lasers for telecommunications," The 15th Annu. Meeting IEEE Lasers and Electro-Optic Society (LEOS), vol. 2, pp. 538-539, Nov. 2002.

[10] J. T. Getty, L. A. Johansson, E. J. Skogen, and L. A. Coldren, "1.55- $\mu \mathrm{m}$ bipolar cascade segmented ridge lasers," IEEE J. Sel. Topics Quantum Electron., vol. 9, no. 5, pp. 1138-1145, Sep./Oct. 2003. 
[11] S. G. Patterson, G. S. Petrich, R. J. Ram, and L. A. Kolodziejski, "Continuous-wave room temperature operation of bipolar cascade laser,” Electron. Lett., vol. 35, no. 5, pp. 395-397, Mar. 1999.

[12] T. B. Simpson, J. M. Liu, and A. Gavrielides, "Bandwidth enhancement and broadband noise reduction in injectionlocked semiconductor lasers," IEEE Photon. Technol. Lett., vol. 7, no. 7, pp. 709-11, Jul. 1995.

[13] T. B. Simpson, J. M. Liu, K. F. Huang, K. Tai, C. M. Clayton, A. Gavrielides, and V. Kovanis, "Cavity enhancement of resonant frequencies in semiconductor lasers subject to optical injection," Phys. Rev. A, vol. 52, no. 6, pp. R4348-51, 1995.

[14] X. J. Meng, T. Chau, and M. C. Wu, "Improved intrinsic dynamic distortions in directly modulated semiconductor lasers by optical injection locking," IEEE Trans. Microw. Theory Tech., vol. 47, no. 7, pp. 1172-1176, Jul. 1999.

[15] A. Murakami, K. Kawashima, and K. Atsuki, "Cavity resonance shift and bandwidth enhancement in semiconductor lasers with strong light injection," IEEE J.
Quantum Elect., vol. 39, no. 10, pp. 1196-204, Oct. 2003.

[16] L. Chrostowski, X. Zhao, and C. J. Chang-Hasnain, "Microwave Performance of Optically Injection-Locked VCSELs," IEEE Trans. Microw. Theory Tech., vol. 54, no. 2, pp. 788-796, Feb. 2006.

[17] H. K. Sung, E. K. Lau, and M. C. Wu, "Optical Properties and Modulation Characteristics of UltraStrong Injection-Locked Distributed Feedback Lasers," IEEE J. Select. Topics Quantum Elec., vol. 13, no. 5, pp. 1215-1221, Sep./Oct. 2007.

[18] S. Mohrdiek, H. Burkhard, and H. Walter, "Chirp reduction of directly modulated semiconductor lasers at $10 \mathrm{~Gb} / \mathrm{s}$ by strong $\mathrm{CW}$ light injection," J. Lightwave. Tech., vol. 12, no. 3, pp. 418-24, Mar. 1994.

[19] H.-K. Sung, T. Jung, M. C. Wu, D. Tishinin, K. Y. Liou, and W. T. Tsang, "Optical Injection-Locked GainLever Distributed Bragg Reflector Lasers with Enhanced RF Performance," International Topical Meeting on Microwave Photonics (MWP), pp. 225-228, Sep. 2004. 